\title{
A Method of Evaluating the Happy Society
}

\author{
Ziqi Wang \\ North China Electric Power University, No.2 Beinong Road, Changping District, Beijing, China \\ axxdwzq@163.com
}

Keywords: The Evaluation Model of Happiness, Structural Equation Modeling, Neural Network

\begin{abstract}
As we can see, the earth on which human beings live has been consumed excessively. It would be better if we could look for a replacement for the earth. According to the current research data show that Mars is more likely for mankind to migrate. Imagine if one day humans succeed in moving to Mars, then how to build a stable and happy Mars society? In this paper, by using the control variable algorithm and structural equation, we establish a Mars social evaluation model of happiness. We use the control variable set algorithm. We set up the evaluation parameters, such as hourly wage, working hours, professional skills, the institution of social compulsory education, maternity leave, social security institution, which are considered from the perspective of three priority factors (income, education, equality). We use the structural equation as the foundation through the Amos platform, to build a sense of happiness evaluation model. Then, with the help of data on GDP output of the provinces in China in 2016, we use BP neural network to analyze the amount of fixed assets, population and try to find their impact on GDP. Meanwhile, by using the method of relative weighting, the normalization of the sense of happiness and GDP is made to measure the sustainable development of Mars society. Our model can consider and deal with multiple dependent variables simultaneously, and present a clear influence of data on the evaluation model.
\end{abstract}

\section{Introduction}

Mars is not only an object to explore and research, compared with other known extraterrestrial planets, but also a magical world. The presence of liquid water and other resources on Mars allows immigrants to grow food, produce plastic and metal, and produce a lot of energy .All elements that human society use a lot on earth, can be found with abundant reserves on Mars. The environmental conditions of Mars can be evaluated from radiation, available sunshine, the temperature difference between day and night, and those aspects are also fit in human suitable survival land surface tolerance range. The energy of Mars makes the red planet likely to be a new life community of millions of residents, in other words, it will create a new world.

Under the premise that Mars is suitable for human migration, it is necessary for us to seek a set of mathematical and computational model. Through this model to develop Mars society operation policy, realize the task of successful human migration to Mars, eventually creating a sustainable society on Mars.

Constructing a social model of sustainable development is the target of our work. Consider the three essential sections of society: the economy, the education system, the workforce,and thequantifiable indicators corresponding to the above three sections are income, education, and social equality. In order to establish a perfect social model:

Firstly for quantitative index we define parameters in more detail, such as the social security institution, social education institution, and maternity leave.

Secondly, we determine from GDP and the sense of happiness of two large aspects to evaluate the Mars society. Through the establishment of a happiness structural equation, a GDP neuralnetworkalgorithmandtheequationaboutthetwocomprehensive,wecanquantitativelyevaluatethe Mars Society. 


\section{Assumptions}

Ignore the differences in the contribution of different ethnic groups to GDP. Our model basedon the data of China Statistical Yearbook to explore the impact factors of GDP, and we thinkthat the ability of different ethnic groups to create GDP was not significantly different.

Ignore the natural conditions of Mars on the impact of the social operation of Mars.We explorethe impact of Mars Society, there is a major premise that Mars is in good condition, naturaldisasters will not occur.

\section{Model}

1. The Establishment of Model[1]. For happy life, we can evaluate it from the two aspects of objective and subjective. To measuresubjective happiness, there are obvious and latent variables. Obvious variable refers to reflect thestatus of happiness through an index. Latent variable refers to the sense of happiness is subdividedinto several aspects, and each aspect selects some indicators to reflect. This analysis method canobtain the cognitive experience, and get a more stable attitude and intention of the information.In the analysis of the influence factors of happiness, we mainly focus on the income, education andsocialequality.We first set up a structural equation model of happiness, then use BP neuralnetwork. Finally unite indicators reasonably, carrying on the analysis to the successful society.

2 .A Structural Equation Model of Happiness[2]. In the evaluation model, we use the structural equation to deal with such a multi-dimensional problem. The structural equation model integrates factor analysis, path analysis. Our model includes: (1) measurement, reflection the relationship between latent variables and measurement index; (2) structure, reflect the structural relationships between latent variables and latent variables.

In our structural equation, the parameters we need to estimate include:

(1) Structural equation coefficients of exogenous latent variables and endogenous latent variables;

(2) The measurement equation coefficient for identifying variables and latent variables;

(3) Error estimation (reflect the size of remaining error);

(4) Estimation of covariance between error term and error term (reflect the association between identifier variables);

(5) The variance of exogenous latent variables. In this paper, the likelihood ratio chi square test is used to test the goodness of fit of structural equation model.

We believe that the income, education and social equality will have a certain impact on personal happiness. The question we are looking at is the intensity of the impact. Whether it is conducive to steady development of the Mars society.

Structural equation models usually contain three matrix equations:

$$
\begin{gathered}
y=\wedge_{y} * \eta+\varepsilon \\
x=\wedge_{x} \xi+\delta \\
\eta=\beta \eta+\Gamma \xi+\zeta
\end{gathered}
$$

$\wedge_{\mathrm{x}}$ : The direct relationship between exogenous variables and exogenous latent variables

$\wedge_{\mathrm{y}}$ : The direct relationship between endogenous variables and endogenous latent variables

$\beta$ : path coefficient of endogenous latent variables

$\eta$ : path coefficient between exogenous latent variables and endogenous latent variables

$\xi$ : the residual term of structural equation

$\varepsilon, \delta, \zeta$ :error term

We determine the impact factors affecting the happiness and its parameters, through the Amos software to build the structure chart:

Three indicators $\mathrm{X}_{1}, \mathrm{X}_{2}, \mathrm{X}_{3}$ of income: hourly wage, working hours, income distribution 
institution

Two representative indexes $\mathrm{X}_{4}, \mathrm{X}_{5}$ of education: professional skills, the institution of social compulsory education

Two indicators $\mathrm{X}_{6}, \mathrm{X}_{7}$ of equality: maternity leave, social security institution

$\mathrm{X}_{8}, \mathrm{X}_{9}$ : the influence of work on happiness, the influence of life on happiness

The Structural Equation of Happiness

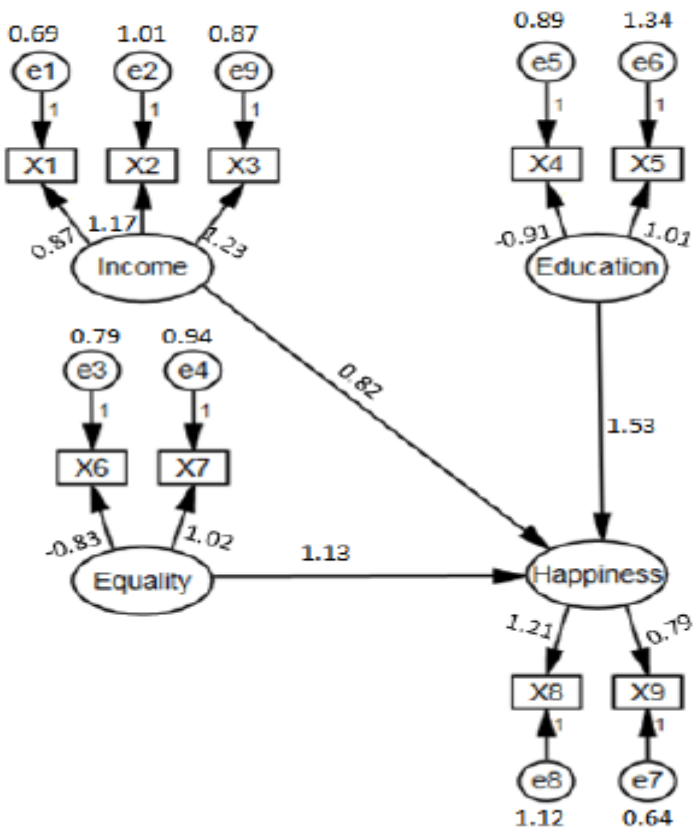

Fig. 1The Structural Equation of Happiness

What can be calculated using AMOS:

Regression Weights: (Group number 1 - Default model)

\begin{tabular}{|c|c|c|c|c|c|c|}
\hline & & & Estimate & S.E. & C.R. & P Label \\
\hline Happiness & $<--$ & Education & -.135 & .059 & -2.270 & .023 \\
\hline Happiness & $<---$ & Income & .406 & .098 & 4.154 & $* * *$ \\
\hline Happiness & $<---$ & Equality & -44.527 & 294.189 & -.151 & .880 \\
\hline $\mathrm{X} 1$ & $<---$ & Income & 1.000 & & & \\
\hline $\mathrm{X} 2$ & $<---$ & Income & .586 & .128 & 4.565 & $* * *$ \\
\hline $\mathrm{x} 6$ & $<---$ & Equality & 1.000 & & & \\
\hline$x 7$ & $<---$ & Equality & -41.889 & 276.759 & -.151 & .880 \\
\hline $\mathrm{X} 4$ & $<---$ & Education & 1.000 & & & \\
\hline X5 & $<---$ & Education & 1.666 & .973 & 1.712 & .087 \\
\hline X9 & $<---$ & Happiness & 1.000 & & & \\
\hline $\mathrm{X} 8$ & $<---$ & Happiness & 1.321 & .184 & 7.167 & $* * *$ \\
\hline $\mathrm{X} 3$ & $<---$ & Income & .941 & .182 & 5.162 & $* * *$ \\
\hline
\end{tabular}

Fig. 2Operation Result on AMOS

Through the structural equation model of happiness, we can easily get the parameters that we need. Model test results: (We use GFI and RMR acceptance standard)

It shows that: 
RMR, GFI

\begin{tabular}{|l|rrrr|}
\hline Model & RMR & GFI & AGFI & PGFI \\
\hline Default model & .416 & .805 & .650 & .447 \\
Saturated model & .000 & 1.000 & & \\
Independence model & .525 & .526 & .408 & .421
\end{tabular}

Fig. 3Model Test Result

The education level has a significant positive impact on the happiness. In fact, in the relationship between educational level and happiness, it's more likely for well-educated to obtain happiness. Social security also has a positive effect on happiness. This means that the people satisfaction of social security is not high, the happiness will reduced. Shown in the figure, the social security factor on the happiness of the impact is significantly greater than the income distribution factor. We believe that the income distribution reflects the reconstruction of interest mechanism, in this process there are people benefit, people who benefit damage, mutative sense of happiness is normal. Social security is related to people's life, if social security coverage is small, or social security is too low, not enough to make people live and work in peace, is bound to reduce people's happiness. The impact of income on happiness is that, the lower the distribution of income satisfaction is, the lower the people's sense of happiness is. In fact, there is interaction between the groups, such as high income, low educated young people's happiness; low income, highly educated young people's happiness. The mesh influence between multidimensional factors can be analyzed through Amos.

3. Analysis of Influencing Factors of GDP. The analysis and forecasting of GDP is of great importance to economic development and social development. This essay, taking China's various regions as an example, analyzes and validates the three factors discussed above, through the method of BP neural network. The impact of GDP should be multi-faceted. For the direct impact of GDP factors, we considered Gross Fixed Capital Formation $\left(\mathrm{X}_{1}\right)$, Gross Regional Product $\left(\mathrm{X}_{2}\right)$, and Total Population $\left(\mathrm{X}_{3}\right)$. Based on these three factors, a prediction model is established.

GDP (gross domestic product) is a measure important indicator of national economy. The three common factors that affect the $\mathrm{GDP}(\mathrm{Y})$ are the gross fixed capital formation $\left(\mathrm{X}_{1}\right)$, the gross regional product $\left(\mathrm{X}_{2}\right)$, and the total population $\left(\mathrm{X}_{3}\right)$, which are used in migration program. Education determines the economic base of a region and plays a decisive role in economic development. From the China Statistical Yearbook, we can obtain these data. From China's GDP calculation formula, we identified these three main factors. Assume that GDP and the three factors satisfy the following functional relationship:

$$
Y(G D P)=F\left(X_{1}+X_{2}+X_{3}\right)
$$

The function relation is constructed by BP network method, and we test our analysis.

4. Analysis Based on BP Neural Network[3]. First of all, BP network is established. In this paper, three-layer network structure is adopted. Theinput layer uses the above three factors $\mathrm{X}_{1} \mathrm{X}_{2} \mathrm{X}_{3}$ as the input node, the output layer uses the GDPvalue $\mathrm{Y}$ as the output node. The three indicators as the value of the network input into the establishment of the BP network for training. Through the comparative training,the number of nodes in the hidden layer is 9, and the transfer function of "s" is set and "pure linear" istaken as the transfer function of the output layer. Using MATLAB for calculation, finally, at the step135, the ideal fitting results come out.

The success of network training shows that there is a functional relationship between GDP and threefactors:

$$
Y=2867.6205+0.1225 X_{1}+0.5309 X_{2}+0.7147
$$




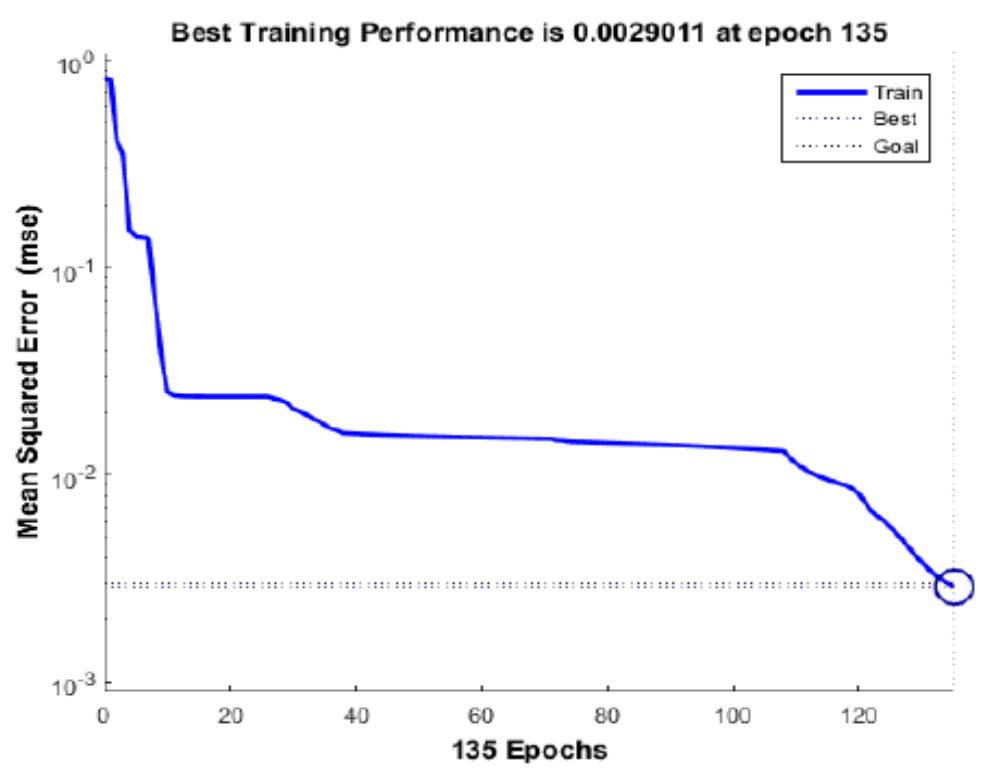

Fig. 4Error Test

In order to verify the effectiveness of using the BP neural network designed approximation function, we use the Postreg Function in MATLAB to make linear regression analysis between the actual output and the ideal output of the network training samples. The correlation coefficient $\mathrm{R}=$ 0.99195 of the ideal output to the actual output of the network was obtained, which indicated that the training result was very good. The results are as follows:

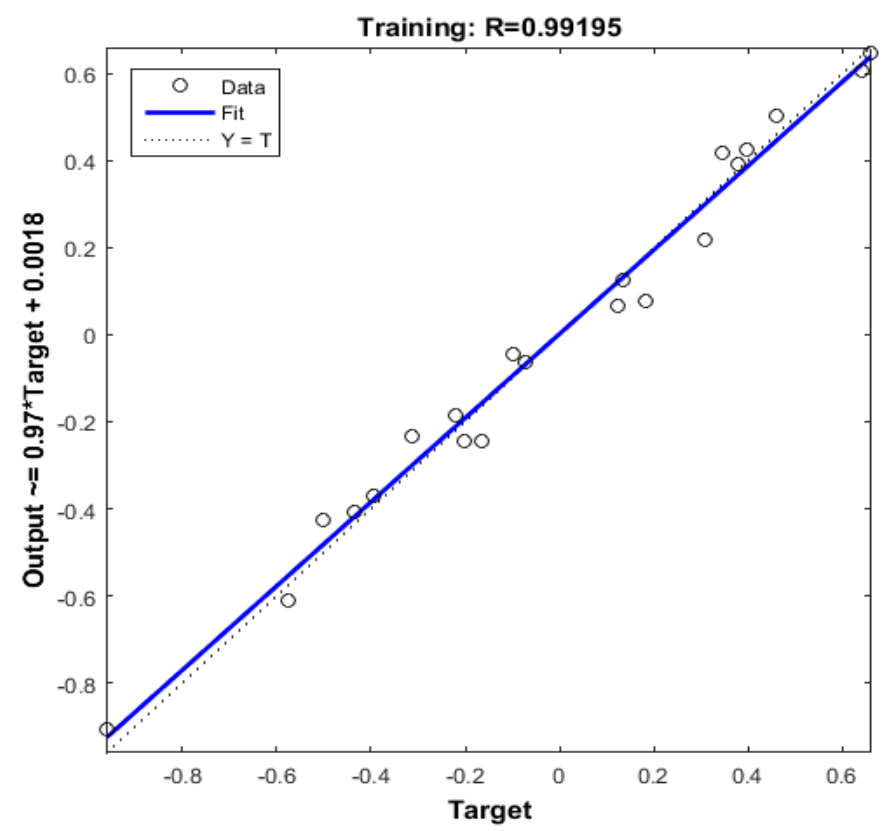

Fig. 5Fit Test

Through BP neural network, we conclude that the gross fixed capital formation (X1), the gross regional product (X2), and the total population (X3) are the main factors affecting GDP. We can also establish fitting function to predict GDP.

5 . Integrated Model. The relative weighting method is used to compute the weights of GDP and happiness. The evaluation indexes are arranged in rows and columns to form a square table. The relative importance of any two indexes is analyzed according to the three-level scale, and the scores are recorded in the corresponding positions in the table. The scores were summed according to the line, and the scores of each index were summed up. Finally, the normalization processing was done to get the weight coefficient of the index.[4]

With a scale of 1-9:

1) Psychological experiments show that it reflects the majority of people's ability to judge; 
2) Social survey shows that the proportion of scale has been used by people adroitly;

3) Scientific investigation and practice shows that the proportion of scale has been able to distinguish between sensory differences caused by the various properties of things.

Personal perspective: According to China Yearbook analysis, happiness is more important than GDP, the scale a12 $=7$.

Social point of view: According to the Chinese Yearbook, the government thinks that GDP is more important than happiness, the scale a21 $=5$.

For a stable development of society, social point of view is more important than personal, more conducive to the harmonious development of society. So the scale a00 $=5$.

We can get that GDP final score is of 11 scores, the final score of happiness is 9 scores.

Normalized processing made the weight of GDP is 0.55, the weight of happiness is 0.45.It can be obtained that GDP is more conducive to promoting the development and progress of Mars society, maintaining the social harmony of Mars.

6. Main Advantages and Disadvantages of the Model

Structural equation analysis can consider and deal with multiple dependent variables simultaneously. In regression analysis or path analysis, even if multiple dependent variables are displayed in the graph of the statistical results, the regression coefficient or path factor is calculated for each dependent variable. So the chart appears to consider more than one dependent variable, but in the calculation of a dependent variable of the impact or relationship, have ignored the existence of other dependent variables and their impacts.

The structural equation analysis allows the independent and dependent variables to contain measurement errors. Variables can also be measured with multiple metrics. The correlation coefficient between the latent variables calculated by the traditional method and the latent variable calculated and analyzed by the structural equation may vary widely.

The traditional factor analysis is difficult to deal with a model that has multiple complex factors such as a subordinate factor or a higher order factor, but structural equation analysis allows for more complex models.

Estimating the degree of fitting the entire model in the traditional path analysis, we only estimate the strength of each path (the relationship between variables). In structural equation analysis, in addition to the estimation of the above parameters, we can also calculate the different models on the same sample data, the overall degree of fitting to determine which model is closer to the data presented by the relationship.

\section{References}

[1] Z.B. Li, L.Wang, H.S. Chen, S.C. Zhu. Establishment and Discussion of Comprehensive Evaluation and Quantitative System of Well-being [J] .Second Science Monthly, 2011, (11): 103-104. (In Chinese)

[2] S. Feng. Structural equation model of subjective well-being [J]. Statistical Research, 2007, (02): 27-32.(In Chinese)

[3] L.J. Qiu, C.Z. He. Cross-validation Model for Stability of Neural Networks [J].Computer Engineering and Applications, 2010, (34): 43-45. (In Chinese)

[4] W.G. Li, C.H. Yu. Study on the Relationship between Per Capita GDP and Happiness Index [J] .Chinese Economic Journal, 2013, (11): 12-13. (In Chinese) 